\title{
American Association of Pharmaceutical Scientists (AAPS) and Chinese National Institutes for Food and Drug Control (NIFDC) Joint Workshop on Dissolution, Bioequivalence, Product Performance, and Quality
}

\author{
Xujin Lu ${ }^{1 *}$, Baoming Ning ${ }^{2}$, Nikoletta Fotaki ${ }^{3}$, Sandra Suarez Sharp ${ }^{4,5}$, Diane Burgess ${ }^{6}$ and Samir \\ Haddouchi $^{7}$ \\ ${ }^{1}$ Bristol Myers Squibb Company, New Brunswick, NJ, USA. \\ ${ }^{2}$ National Institutes for Food and Drug Control, Beijing, China. \\ ${ }^{3}$ University of Bath, Bath, UK. \\ ${ }^{4}$ Center for Drug Evaluation and Research, Food and Drug Administration, Silver Spring, Maryland, USA. \\ ${ }^{5}$ Current: Simulations Plus Inc., Lancaster, CA, USA. \\ ${ }^{6}$ University of Connecticut, Storrs, CT, USA. \\ ${ }^{7}$ SPS Pharma Services, Orléans, France.
}

e-mail:xujin.lu@bms.com

\section{INTRODUCTION}

he second joint workshop sponsored by the American Association of Pharmaceutical Scientists (AAPS) and the Chinese National Institutes for Food and Drug Control (NIFDC) took place in Yantai, China, from May 31 to June 1, 2019. The theme of the workshop was Dissolution, Bioequivalence, Product Performance, and Quality.

Following the success of the first AAPS/NIFDC cosponsored workshop in 2016, this workshop aimed to meet the strong demand from local communities by addressing the following objectives: (1) enhance the communication among Sino and American regulators, academics, and industry scientists in the field of in vitro drug release; (2) share knowledge in dissolution methodology, new apparatus and technology, novel drug formulation performance, and drug specification settings; (3) disseminate regulatory and compendia information to facilitate method development, current Good Manufacturing Practice (cGMP) compliance, and quality control of drug products; and (4) facilitate networking and collaboration among scientists from the drug control and inspection agencies, academia, and the pharmaceutical industry.

The workshop was organized by AAPS In-Vitro Release and Dissolution Testing (IVRDT) Community, Chinese Journal of New Drugs, and Shandong Institute for Food and Drug Control. This event was supported by the Medical Instrument Branch of China Instrument and Control Society, State Key Laboratory of Long-Acting and Targeting Drug Delivery System, Yantai Institute for Food and Drug Control, the Chinese Journal of Pharmaceutical Analysis, and the School of Pharmacy at Yantai University.

\section{SESSION ONE}

The Chair of the Organizing Committee, Dr. Xujin Lu (Bristol Myers Squibb, USA) welcomed participants and gave an introduction to the motivation and aims of the workshop, referencing the previous workshop. In the opening session, the workshop started with dissemination of regulatory guideline and perspective on drug product quality and dissolution testing. Dr. Sandra Suarez Sharp (United States Food and Drug Administration [FDA]) delivered the first lecture entitled "The Role of Biopharmaceutics Tools in Support of Drug Product Quality and Regulatory Approval: FDA Perspective." She emphasized that biopharmaceutics ensures that drug release and absorption from the drug product results in optimal therapeutic efficacy and safety for the patient. Tools used in biopharmaceutics such as bioavailability (BA) and bioequivalence (BE) measures are employed to provide a linkage between changes in the critical material attributes (CMAs), critical process parameters (CPPs), and clinical outcomes supporting patient-centric drug product quality. Without a dissolution method that increases the likelihood of developing a successful in vitro-in vivo 
correlation/in vitro-in vivo relationship (IVIVC/IVIVR), the burden for establishing this linkage becomes very high, making it considerably expensive, time consuming, and impractical. Thus, the development of a dissolution method that is not only discriminating, but also clinically relevant, becomes critical, especially for immediate release drug products containing Biopharmaceutics Classification System (BCS) class II and IV drugs and modified release drug products.

The application of IVIVC in drug development to obtain biowaivers and setting dissolution specifications for solid dosage forms is well recognized. The role of IVIVC and IVIVR in the Quality by Design (QbD) paradigm has been further emphasized to enhance drug product understanding and setting clinically relevant drug product specifications. IVIVC also provides a pathway for linking product attributes to clinical safety and efficacy. However, the success rate of conventional IVIVC establishment is still low in regulatory submissions, which greatly discourages sponsors to conduct IVIVC studies. Dr. Suarez Sharp also mentioned that one of the primary reasons for the low rate of success could be attributed to the conventional methodologies currently used for developing IVIVCs. She stated that these methodologies may not meet the needs for IVIVC establishment due to insufficient considerations on the in vivo dissolution and absorption mechanisms under physiological state. In addition, IVIVC guidance recommends the construction of IVIVCs studies to be conducted under fasting conditions, limiting its implementation to drug products whose BA is not impacted by food. The advanced modeling approach, namely physiologically based biopharmaceutics model (PBBM) shows promising advantages in increasing the success rate in building IVIVRs/IVIVCs. This can be attributed to the possibility to incorporate different mechanisms influencing drug disposition and absorption (e.g., drug release, gastrointestinal (GI) transition, metabolism, and elimination) and provide more realistic perspectives regarding the effect of formulation and manufacturing changes on in vivo performance. Recently, PBBM has been increasingly used in regulatory submissions as important tool in drug development and regulatory review for various designed purposes, e.g., the development of clinically relevant specifications, the design of IVIVC in a mechanistic framework, and in support of biowaiver request. Nevertheless, there are still several challenges in the implementation of PBBM due to gaps in knowledge including the identification of best approaches to integrate manufacturing changes, criteria for model validation, and the use of virtual $\mathrm{BE}$ analysis.
As Dr. Suarez Sharp mentioned above, in vivo studies measuring the $\mathrm{BA}$ and $\mathrm{BE}$ during drug development play a pivotal role such as bridging major formulation/ manufacturing changes. For orally administered drugs, the in vivo $B A / B E$ study requirement, in certain instances, can be waived provided the following requirements are met: (1) the applicant followed the provisions outlined in regulations, 21 CFR 320.22(d) (2); (2) the requirements outlined under the BCS guidance are met; (3) a safe space is built via mechanistic/conventional IVIVR/IVIVC; and (4) a risk assessment approach is used. When it comes to implementing a risk assessment approach, it may be feasible if, in certain situations, the intricacy of the proposed changes may not fulfill the biowaiver criteria (e.g., compositional nonproportionality). Such scenario calls for considerations of relevant in vitro/in vivo data collected during drug product development and riskbenefit assessment to judge the clinical impact of not meeting the required criteria.

In conclusion, Dr. Suarez Sharp emphasized that biopharmaceutics tools, such as dissolution testing, play a key role in supporting drug product development and in the approval of drug products throughout the lifecycle. Thus, efforts should be invested in developing dissolution methods that are clinically relevant with the purpose of increasing the success rate of IVIVC/IVIVR, which are essential approaches for building a safe space. Building a safe space is not only relevant for gaining regulatory flexibility but is also a stepping-stone toward patientcentric drug product development.

\section{Professor Yamin Wang (Center for Drug Evaluation} [CDE], China) gave the second talk, entitled "Dissolution Methodology and Quality Evaluation of Solid Oral Dosage Drug Products: CDE Perspective." Professor Wang first introduced the CDE and the rationale of the Chinese guidance for BE evaluation of generic drugs, including the definition of drug dissolution and its function in solid oral drug quality evaluation. He indicated the importance of inclusion of dissolution test and study in common technical document (CTD) documentation and submission, and pointed out that the agency paid more attention to the discriminating ability of the dissolution conditions. A discriminatory dissolution method should be able to identify the changes of drug product quality caused by changes in formulation and manufacturing process, especially for the changes affecting the biopharmaceutics performance. He further elaborated in details with examples for the consideration of conducting dissolution study for both innovative drug development of and generic drug development. Professor Wang finally 
emphasized that dissolution performance is critical to drug product evaluation and quality control; it can be predictive of drug BA. Currently, efforts have been made to connect the Chinese guidance for drug evaluation with an international standard system. He encouraged paying more attention on dissolution method condition selection and validation, dissolution profile comparison, setting appropriate dissolution specification. He also welcomes communication and dialog between the industry and the CDE agency.

The trends and compendial requirements on dissolution methodologies and apparatus were also addressed in this section. Dr. Baoming Ning (NIFDC, member of Chinese Pharmacopoeia Expert Committee) gave a talk on "Overview and Dynamics of Dissolution Technology in China." Dr. Ning first gave an introduction of NIFDC, including its history, organization structure, responsibility, and international cooperation. Then, Dr. Ning reviewed the theory, origin, and practice of dissolution technology and related domestic and international activities. He highlighted the seven compendial dissolution apparatus, followed by introduction of the dissolution monographs in Chinese Pharmacopoeia (ChP), the recorded apparatus and the evolution of dissolution devices made in China. Dr. Ning presented updated information on the evaluation of United States Pharmacopoeia (USP) apparatus 3 and 4 from ChP. He indicated that both USP 3 and 4 apparatus are in consideration by ChP to be adopted in its 2020 edition. Dr. Ning's team is supported by ChP for evaluation and application of the USP apparatus 3, 4, and 7. He presented the progress and recent accomplishment in applying these apparatus on drug product quality, IVIVC, and BE studies. Through an example of felodipine sustained release tablets, he also addressed the importance of establishing a biorelevant and discriminating dissolution method using these apparatus.

Dr. Diane J. Burgess, distinguished Professor (University of Connecticut, USA) gave a lecture on "Application of USP Apparatus 4." This lecture focused on the application of USP Apparatus 4 to the in vitro release testing of complex parenterals, such as liposomes, microspheres, and ophthalmic ointments. USP apparatus 4 (flow-through cell) was originally designed for the in vitro release testing of controlled release oral products. It was shown by comparison with other methods such as USP apparatus 2 and Franz cells that USP apparatus 4 was an excellent method for in vitro release testing of complex parenterals such as liposomes, microspheres, and ophthalmic ointments. The attributes of the various compendial and noncompendial apparatus were first discussed and then results were given for three cases studies on Risperidal Consta microspheres, dexamethasone liposomes, and Loteprednol Etabonate ophthalmic ointments. It was shown that USP apparatus 4 was the most attractive method for all three complex parenteral formulations and was able to discriminate under both "real-time" and accelerated conditions, compared to USP apparatus 2, dialysis sac, reverse dialysis sac, and Franz cell methods. For the ophthalmic ointments, it was shown that the drug release was correlated to the rheological properties (storage modulus, loss modulus, onset point, and crossover modulus). The in vitro release from the ophthalmic ointments was also shown to correlate with the transcorneal flux from the formulations.

Dr. Nikoletta Fotaki (University of Bath, UK) gave a presentation entitled "Application Case Studies of USP Apparatus 4." She discussed initially the main characteristics and the key features of the USP apparatus 4. Points to consider for the development of dissolution methods with the use of this apparatus in terms of hydrodynamics were also noted. The case studies covering a wide range of applications of the USP apparatus 4 included: (1) the effect of glass bead size on drug dissolution; (2) the effect of media and hydrodynamics on release from parenteral suspensions; (3) the use of USP apparatus 4 as a tool for setting conditions to ensure consistent in vitro/in vivo performance of oral drug products; (4) for predicting the effect of food; (5) for investigation of the dissolution mechanism of poorly soluble drug and conformers from pharmaceutical cocrystals; and (6) for the development of IVIVC for an amorphous formulation of a poorly soluble compound. Overall, the importance of selection of operational variables, media and hydrodynamics for the development of the USP apparatus 4 release/dissolution method and its successful use as a quality tool for the assessment of BA during formulation screening, the characterization of solid oral dosage forms and parenteral suspensions, and the development of IVIVCs were revealed.

\section{SESSION TWO}

The second session began in the afternoon with Dr. Xujin Lu giving an overview of the AAPS and the AAPS IVRDT community, the US cosponsor and organizing institution of the event. He also highlighted that Chinese scientists have access to the AAPS international and China Chapter memberships.

The theme of this session focused on imaging assistant characterization of drug release and dissolution studies, with emphasis on the studies of drug release 
mechanism and dosage form performance that have been significantly enhanced by implementation of new technologies. Dr. Zhen Liu (Merck, USA) presented the first talk titled "Application of 3D Image Technology in Drug Development." The 3D imaging technologies presented is a combination of X-ray computed tomography (CT) and Focused Ion Beam Scanning Electron Microscopy (FIB-SEM). X-ray CT is a nondestructive technique for visualization of interior features of solid objects, and for obtaining digital information on their 3D geometries and properties. A CT image slice corresponds to a certain thickness of the object being scanned. By stacking all of CT slice images, a complete volumetric representation of an object is obtained via acquisition of a contiguous set of CT slices. FIB-SEM uses a finely focused beam of ions for site-specific milling and a focused beam of electrons to image the sample. A correlative imaging obtained through combination of the aggregate scale representativeness (Micro-CT) and primary particle scale (FIB-SEM) provides more accurate microstructural analysis for simulating drug release performance. Dr. Liu introduced a correlative multi-scale image based drug characterization workflow for characterization of controlled release and extended release dosage forms. This workflow includes three steps: imaging, analytics, and simulation. Through the workflow, high resolution images can reveal critical difference in microstructure with unprecedented detail. Quantifications of the microstructure, in combination with computational fluid dynamics (CFD) simulations of drug release process, can successfully predict the drug release behavior of products. All were demonstrated with Implanon (an etonogestrel implant). Dr. Liu concluded that the combination of advanced imaging, automated image analytics, and image-based numerical modeling opens a new frontier in the analytics of drug research and development. In addition to controlled release drug delivery, the digital imaging based characterization approach has potentially broad applications in early and improved in the following areas: pharmacokinetics evaluation; chemistry, manufacturing, and control (CMC) manipulation; and manufacturing quality assurance (QA).

Ms. Liping Zhou (DigiM, USA) was the next presenter. Her talk was on "Key Framework Technologies in the Characterization and Optimization of Microstructures in Drug Release System." Characterization of dosage forms, especially their microstructures, can directly facilitate mechanistic understanding of drug release behavior and improve formulation design and development. Dr. Zhou first introduced the collaborated works that DigiM with a several pharmaceutical companies in combining microimaging and image-based simulation for evaluation and predicting the performance of drugs and different formulations. She then discussed two case studies.

The first case study was on the microimaging characterization and release prediction of control-release Arestin microspheres, to explore the influence of internal microstructures of poly lactic-co-glycolic acid (PLGA) microspheres on drug release with high-resolution imaging and image-based analytics. The method used in the study was FIB-SEM. Two formulations of the microspheres with different release rates were characterized through the imaging, active pharmaceutical ingredients (API), polymer, drug/polymer interface porosity, and polymer porosity clearly identified. 3D images were reconstructed for the drug, as well as for the pores. The effective diffusivity of drug release was calculated, and the release profiles were predicted through the image-based numerical simulation.

The second case study was on noninvasive quantitative characterization of lyophilized drug product using 3D $\mathrm{x}$-ray micro-computed tomography (microCT) (3D XRM) and artificial intelligence (AI))-based image processing and advanced image quantification. A protein-lyophilized cake was scanned in a glass vial as well as taken out from the vial. The cross sections of 3D XRM scans showed clear differences in the cake solid phase and air phase. Imaging and quantification results from using instruments with different resolutions were compared. Changes reflecting the cake wall deformation due to absorbing environment moisture during a few hours of XRM scan were also observed. The studies have demonstrated that the 3D XRM imaging is capable of characterizing the soft solid that is not suitable for certain studies.

Dr. Xujin Lu presented a talk titled "Application of Spectroscopic Imaging Technologies to Dissolution and Formulation Development." Dr. Lu pointed out that the detection of drug release in dissolution testing has been improved through two revolutions. The first one was the use of ultraviolet (UV) fiber optic spectroscopy for in situ measurement of released drug in dissolution vessel without sample removal. The in situ UV measurement enabled generation of detailed dissolution profiles with frequent data points and improved laboratory productivity. The second revolution to dissolution testing was the application of spectroscopic imaging technologies, which enabled the study of dissolution mechanisms and observation of dynamic dissolution process in situ with high spatial and temporal resolution. Spectroscopic imaging measures the changes on or close to the surface of the solid sample or tablet. It provides information on the changes of chemical and physical properties of drugs and 
excipients that are relevant to dissolution mechanisms. This revolution was achieved through a combination of advancements in spectroscopies, instrument hardware, imaging detectors, and data processing techniques. Dr. Lu introduced the characteristics of the major spectroscopic imaging technologies for dissolution applications, including nuclear magnetic resonance (NMR), Raman, Fourier-transform infrared (FTIR), nearinfrared (NIR), and UV. These technologies have attracted much attention not only in academic, but also in industry. These technologies have been applied for understanding of drug release mechanism, formulation comparison, selection, and troubleshooting. UV dissolution surface imaging has been commercially available among these spectroscopy techniques for dissolution imaging. In comparison to the others, UV surface dissolution imaging costs less, but is easier to operate and data processing is less complicated. Dr. Lu presented three application case studies. The first case study was on the selection of carrier for coprecipitated API. Multiple prototype formulations of the API coprocessed with different carriers were studied using UV dissolution imaging on compact flow cell. A suitable carrier was identified based on the UV imaging of the carrier swelling, API diffusion, and observed intrinsic drug release rate. The second case study was on the development of a lipid tablet. The lipid tablet formulation was developed in order to improve the BA and processing ability of a BCS class II drug with high hydrophobicity. UV dissolution imaging was utilized along with micro dissolution; both are sample scarce methodology, capable for biorelevant media, and suitable for early phase drug product development. The third case study was on whole tablet measurement using UV dissolution imaging with a whole dosage flow cell. Instead of testing a small compact of API or granulation, a large flow cell similar to USP apparatus 4 has been available to allow the UV imaging study of a whole tablet or capsule. This is especially useful for extended release formulation development.

\section{SESSION THREE}

Predictive dissolution, clinically relevant specifications, and their application for formulation development were covered in this session. Dr. Nikoletta Fotaki gave the first presentation titled "In Vivo Predictive Dissolution and IVIVC." She discussed the use of biorelevant dissolution testing during early phases of drug development and also on the development of IVIVCs. The physiological conditions and the physical parameters that can affect drug dissolution and the principles for the design of in vivo predictive tests in terms of media and hydrodynamics were presented. Points to consider for the biorelevant media were emphasized, covering updated versions of biorelevant media as a result of the ongoing research for the characterization of the gastrointestinal contents, the vivo variability, and the use of in-house data built with different media versions and the preparation methods. In terms of the appropriate in vitro hydrodynamics, aspects regarding the selections of the in vitro dissolution apparatus, viscosity and simulation of the in vivo hydrodynamics were described. The classification of the drug substance according to the BCS and the choice of appropriate media and volumes are the first steps for the development of a biorelevant dissolution method. Drug substance properties in terms of solubility would define the choice of the medium. For poorly soluble compounds (BCS class II and IV) the factors influencing dissolution, i.e. pH, buffer capacity, ionic strength, presence of surfactants, and/or food components should be considered. Details on the choice of medium based on the ionization properties of the compound and the prandial state were discussed. Formulation aspects need to be taken into account for the design of in vivo predictive tests. Main points for the development of biorelevant release/dissolution tests for immediate and modified release formulations were presented. Dr. Fotaki then explained the methods used for the development of IVIVCs and how data from the in vivo predictive tests are used in the correlations. An IVIVC study for nifedipine immediate release capsules administered with water and orange juice was presented. She also presented a perspective on the development of predictive in vitro testing for patients with gastrointestinal disease and for pediatrics and elaborated on the development of media simulating the gastrointestinal conditions in these populations. She concluded her talk by emphasizing the importance on the design of dissolution tests according to drug substance properties, formulation properties, and condition studied.

\section{Dr. Sandra Suarez Sharp presented afterwards} on "Establishing Clinically Relevant Dissolution Specifications" with emphasis on data needed and current approaches She highlighted that the implementation of the appropriate drug product specifications is essential in assuring consistent in vitro (e.g., dissolution) and in vivo (e.g., efficacy and safety) performance throughout the product's life cycle. The application of risk-based approaches to the control of product quality based on an enhanced process and product understanding (e.g., $\mathrm{QbD}$ ) is in many cases still the focus on qualitybased risk assessment and control, with insufficient or no consideration of clinical relevance. The design space ranges and, subsequently, drug product specifications are 
in many cases established based on passing a dissolution similarity test and/or dissolution acceptance criteria. In some instances, meeting current specifications may not be sufficient to guarantee equivalent clinical performance of a drug product following manufacturing deviations. She emphasized that without an understating of the relationship between the critical quality attributes and the clinical outcome, drug product specification limits may be overly wide, unnecessarily tight or completely irrelevant to clinical performance. She then mentioned that there are several approaches for establishing clinically relevant drug product specifications (CRDPS), depending on whether in vivo data (i.e., systemic exposure) are available. For instance, for BCS class II containing drug products and modified release drug products, the development of IVIVR or IVIVC either via conventional or "mechanistic" approaches is essential to ensure clinical relevance. These approaches enable dissolution testing to define the boundaries of a safe space, a region within which product variants are bioequivalent. Given that the success rate of conventional IVIVCs appears to be low, FDA encourages the use PBBM to be included in regulatory submissions in support of CRDPS. This approach appears to facilitate the development of safe space via either IVIVR or IVIVC, which are central in establishing CRPDS for high-risk drug products.

In conclusion, advancement on in vivo predictive/clinically relevant dissolution combined with "mechanistic" models such as PBBM provides an opportunity for taking a major step in model-based drug development and to support the establishment of CRDPS along with the possibility of gaining regulatory flexibility.

Mr. Samir Haddouchi (SPS Pharma Services, France) give a presentation on "Application of Dissolution Technology in API Quality Characterization." He showed how the dissolution testing of a simple immediate-release dosage form containing a BCS class I substance can be challenging in obtaining an in vitro profile able to predict the in vivo performance of the dosage form. The main objective of his presentation was to outline that dissolution testing could be an extremely powerful tool to acquire knowledge about pharmaceutical products. He pointed out that unfortunately, dissolution profiles are often used without a complete understanding of their meaning and are often considered only when some of the regulatory agencies require data for a submission or to demonstrate appropriate batch-to-batch consistency. Alternatively, we can use the dissolution technique in order to help us learn more about the properties of the API, the composition of the formulation as well as the route of administration.
The scientist should drive to adapt the testing conditions, keeping in mind the aim of the in vitro dissolution method (e.g., formulation development, IVIVC, or Quality Control). Mr. Haddouchi highlighted that the in vitro profiles can represent either the dissolution rate of the API or the release rate from the finished formulation. A more frequent use of API characterization tools such as intrinsic and apparent dissolution (Eur. Ph. §2.9.29 and 2.9.43, respectively) can be of great help in achieving such a goal. By using these additional dissolution tools, it is possible to investigate the root cause of bioinequivalence and to proceed with a more systematic approach for drug product optimization. He also pointed out that the widely used techniques such as paddle and basket apparatus frequently have limitations when it comes to predicting the in vivo performance of drug product while alternative tools such as the flow through cell, because of the different hydrodynamics provided, may have superior advantages for such prediction.

Professor Qian Feng (Tsinghua University, China) was invited as a speaker but unable to attend this workshop. On his behalf Dr. Chengyu Liu delivered the presentation titled "Impact of Drug-Excipient Molecular Interaction on the Dissolution Performance and Bioavailability of Supersaturable Formulations." With the widespread use of combinatorial chemistry and high-throughput screening in modern drug development, more and more small molecule candidates are poorly water soluble. The low solubility of small molecules leads to a series of problems, such as large oral dose, low BA, strong food or $\mathrm{pH}$ effect, poor compliance of patients, etc. The interaction between drugs and excipients, especially polymers and surfactants, will significantly affect the supersaturation and BA of these poorly-water soluble drugs.

In the case of sorafenib, Professor Qian's team observed that its solubility could be increased approximately 50fold in the coexistence of polyvinylpyrrolidone-vinyl acetate (PVP-VA) and sodium lauryl sulfate (SLS), due to the formation of PVP-VA/SLS complexes at a lower critical aggregation concentration. The enhanced solubility provided a faster initial sorafenib dissolution rate, analogous to a forceful "spring" to release drug into solution, from tablets containing both PVP-VA and SLS. However, SLS appears to impair the ability of PVPVA to act as an efficient "parachute" to keep the drug in solution and maintain drug supersaturation. Using 2D 1H NMR, 13C NMR, and FTIR analysis, he concluded that the solubility enhancement and supersaturation of sorafenib were achieved by PVP-VA/SLS complexes and

Dissolution

MAY 2020 Technologies www.dissolutiontech.com 
PVP-VA/sorafenib interaction, respectively, both through molecular interactions hinged on the PVP-VA VA groups. Therefore, a balance between "spring" and "parachute" must be carefully considered in formulation design. To confirm the in vivo relevance of these molecular interaction mechanisms, the team prepared three tablet formulations containing PVP-VA alone, SLS alone, and PVP-VA/SLS in combination. The USP apparatus 2 in vitro dissolution and canine PK in vivo evaluation showed clear differentiation between these three formulations, as well as good IVIVC. The formulation containing PVP-VA alone demonstrated the best BA with 1.85-fold and 1.79fold increases in Cmax and Area under the curve (AUC), respectively, compared to the formulation containing SLS only, the poorest performing one. Despite its forceful "spring", the formulation containing both PVP-VA and SLS showed a moderate BA enhancement, due to the lack of an efficient "parachute."

In another case for posaconazole (PSZ), this team found that SLS greatly influences the BA of amorphous solid dispersions though it is an effective surfactant, and often used as a solubilizer and/or wetting agent in various dosage forms for the purpose of improving the solubility and dissolution of lipophilic, poorly-water-soluble drugs. Although FTIR study demonstrated the existence of specific interactions between PSZ and hypromellose acetate succinate (HPMCAS) in the solid state, predissolved HPMCAS was unable to maintain the supersaturation of PSZ effectively. The coprecipitated HPMCAS was found to be able to significantly delay the crystallization of PSZ in the PSZ-rich amorphous phase from less than 10 minutes to more than 4 hours. Yet, coexistent SLS was able to negate this crystallization inhibition effect of HPMCAS in the PSZ-rich amorphous precipitates and cause fast PSZ crystallization within 30 minutes. 2D-NOESY and the CMC/CAC results demonstrated that SLS could assemble around HPMCAS and competitively interact with HPMCAS in the solution, thus preventing HPMCAS from acting as an effective crystallization inhibitor. In a crossover canine pharmacokinetic (PK) study, the SLS containing PSZ amorphous solid dispersion (ASD) formulation demonstrated an in vivo BA approximately $30 \%$ of that without SLS, despite the apparently better in vitro dissolution, which only compared the dissolved drug in solution, a small fraction of the total PSZ dose. Thus, the BA of ASDs is highly dependent on the molecular interactions between drug, surfactant, and polymer, not only in the solution phase but also in the drug-rich "oily" phase caused by supersaturation. In summary, Professor Qian's presentation concluded that the interactions between drugs and excipients (such as polymers and surfactant) have a significant impact on the dissolution and absorption of poorly-water soluble drugs. This effect is especially evident when supersaturation exists in the dissolution process.

\section{SESSION FOUR}

The last session of the workshop focused on emerging topics in dissolution. The learning objectives were to recognize the challenges from new drug delivery and new manufacturing process, demonstrate the use of modeling for drug product development and real time release testing (RTRT), and introduce new concepts and methodologies for drug release and performance evaluation.

Dr. Xujin Lu presented the first talk in this session with the title "Predictive and Clinically Relevant Dissolution Models for RTRT in Continuous Manufacturing." Continuous manufacturing has been growing rapidly in pharmaceutical industry. Demands for real time release testing become stronger and more critical in support of such industrial trend. Dr. Lu outlined the role of dissolution in RTRT, the predictive dissolution modeling for RTRT, and the related special challenges. Dissolution is a required and regulated testing for finished drug product quality control and batch release. The nature of the dissolution testing is time consuming, dependent on compendial device and setting, and not suitable for on-line or in-line operation. Instead, predictive modeling has been applied as a surrogate to predict dissolution results for RTRT. In this approach, multivariate models are established based on raw material attributes (RMAs), CMAs, and CPPs that are critical to dissolution and in vivo performance. The models are targeted and validated to a reference dissolution method. The holistic data generated through in-process monitoring and controls are used as the entry to the model to predict dissolution and eventually, to release the final product. Several new drug products approved by FDA have utilized continuous manufacturing with predictive dissolution models for RTRT. Regarding the challenges that such methodologystill faces, Dr. Lu pointed out, firstly there are few successful examples. Secondly, health authorities are encouraging the establishment of clinically relevant dissolution specifications. Yet, the current dissolution modeling for RTRT focuses mainly on in vitro predicting profile or value but does not always address clinical relevance to meet the new regulatory expectation. Recognizing these challenges, many efforts have been made to build a path to clinically relevant dissolution modeling. The International Consortium for Innovation and Quality in Pharmaceutical Development (IQ) Dissolution Working Group, AAPS IVRDT, and 
process analytical technology (PAT) communities have organized forums and workshops to discuss this topic and communicate with regulatory agencies. Dr. Lu elaborated three approaches from the industry point of view: (1) use a clinically relevant dissolution method as the reference method for RTRT modeling; (2) systematically develop a clinically relevant QC dissolution method; and (3) apply $\mathrm{QbD}$ for product design space, develop QC method and dissolution model within the space, and perform PK verification for clinical relevance.

The next speaker, Dr. Nikoletta Fotaki, presented on "PBPK Based Modeling and Simulation in Support of Drug Product Development." She outlined the reasons for attrition in drug development and emphasized the need for the use of accurate tools for the prediction of the in vivo performance. Physiologically Based Pharmacokinetic (PBPK) models are mathematical models that integrate anatomical and physiological parameters of animals or humans, physicochemical properties of drug substances, and formulation properties of drug products. PBPK models are used for the quantitative prediction of a compound's in vivo absorption, distribution, metabolism, and excretion (ADME) by integrating prior knowledge in a mechanistic network. Absorption models (referred as PBBM in Dr. Suarez Sharp's presentation) are a part of whole PBPK models and the combined effects of several processes in the gastrointestinal (GI) tract (such as gastric emptying, transit through the intestinal tract, solubility/dissolution, transport/metabolism through the gut wall) and factors relating to the properties of the drug/formulation have to be taken into account. The input of appropriate in vitro data in the absorption models is essential for the development of successful PBPK models. In vivo predictive design of the in vitro solubility, dissolution, permeability, degradation, metabolism and transporter studies is needed. Dr Fotaki then explored the challenges for the development of PBPK models for the prediction of in vivo performance after oral administration. She presented case studies in which successful IVIVCs were developed based on PBPK modeling in combination with appropriate in vitro conditions. In the first case study, the impact of in vitro dissolution and degradation in the PBPK model for the development of IVIVCs for an amorphous immediate release formulation of a BCS class II compound was shown. In the second case study, she described the development of a PBPK model for the pediatric population for an immediate release formulation of a BCS class II compound, in which the impact of the in vitro dissolution and solubility was explored. The last case study revealed the use of PBPK modeling for the identification of in vivo predictive tests for parenteral liposomal formulations.
Drug release and performance of long acting injectable formulations has attracted much attention in recent years. Dr. Diane J. Burgess presented next with a title of "Drug Release from Long Acting Biodegradable Microspheres of Injectables." Dr. Burgess discussed the development of qualitatively (Q1) and quantitatively (Q2) equivalent formulations of various microsphere drug products (Risperdal Consta and Naltrexone) with manufacturing differences and comparison of their in vitro and in vivo release (rabbit model) together with the release properties of the RLD and the development of suitable level A IVIVC for these Q1/Q2 equivalent formulations. It was demonstrated that the Q1/Q2 PLGA microsphere formulations with manufacturing differences had differences in physicochemical properties such as porosity and particle size and that these differences resulted in differences in both the in vitro and in vivo release of these formulations. Even more importantly, it was demonstrated that IVIVC could be developed for these complex microsphere formulations that involve two and even three different release phases (burst release, lag phase, and secondary release phase). The USP apparatus 4 method was suitable for the development of IVIVCs for the microsphere products. The USP apparatus 4 is known for its effectiveness in suitable method development for controlled release oral formulations (e.g., tablets).

She pointed out that this research is of extreme importance for the development of new complex parenterals to ensure drug product quality and to facilitate regulation of minor manufacturing difference and manufacturing site changes as well as for the development of generic equivalents of complex parenterals.

\section{Dr. Kaoxiang Sun (Luye Pharmaceutical Group, China)} presented on behalf of Prof. Youxin Li (State Key Laboratory of Long-Acting and Targeting Drug Delivery System, Luye Pharm. Group, China). The topic of his presentation was "Application of In Vitro Release Study in the Development of Extended-Release Formulations," which mainly focused on the development of in vitro release method of injectable microspheres. Dr. Sun first introduced the classification of extended-release formulations, the definition, characteristics, release mechanism and attributes of in vitro release profile of microspheres. Then, he described the challenges faced during the development of in vitro release method for microspheres, such as lack of compendial method or apparatus, long release period and complex release mechanism, etc. Dr. Sun compared the flow-through cell (USP apparatus 4) and sampleand-separate method. The former is more suitable for microspheres, while the latter is more common for the 
marketed products. Although accelerated methods of in vitro release can be developed in terms of altering the temperature, $\mathrm{pH}$, organic solvents, surfactants, and/or ionic strength, it is necessary to note that the accelerated method should correlated with the real-time method. At the same time, one should pay attention to the selection of release method in combination with release mechanism. For burst release, the $37^{\circ} \mathrm{C}$ real-time method is more sensitive than the accelerated method. At different $\mathrm{pH}$ values, the release mechanism may change from erosion control to diffusion control. In the case of core-shell microspheres, programmed heating allows full release of PLGA-encapsulated drugs. Finally, Dr. Sun gave an example of how to establish IVIVC for a microsphere formulation and how to set in vitro release specification based on the IVIVC.

\section{SUMMARY}

This second workshop in China, which was sponsored by AAPS and NIFDC, was well attended as the first one, with more than 300 attendees from industry, drug control, and inspection agencies, and academia, including graduate students from local universities (Fig. 1). It provided a great opportunity for direct dialogue between regulatory, industry, and academic stakeholders to identify the gaps in knowledge to move the field forward. The
Q\&A sections were well participated, many attendees actively communicated, and discussed variety topics with the panel of speakers. The attendees expressed strong interests in new regulatory guidance, trends in new technology/methodology, novel applications, and expect that such workshop series can continue to provide educational and regulatory application perspectives.

\section{DISCLAIMER}

This article reflects the views of the authors and should not be construed to represent their organizations' views or policies.

\section{CONFLICT OF INTEREST}

The authors disclosed no conflicts of interest related to this article.

\section{ACKNOWLEDGMENTS}

The authors would like to acknowledge the support from the AAPS, NIFDC, AAPS IVRDT Community, Chinese Journal of New Drugs, Shandong Institute for Food and Drug Control, the Medical Instrument Branch of China Instrument and Control Society, State Key Laboratory of Long-Acting and Targeting Drug Delivery System, Yantai Institute for Food and Drug Control, Chinese Journal of Pharmaceutical Analysis, and the School of Pharmacy at Yantai University.

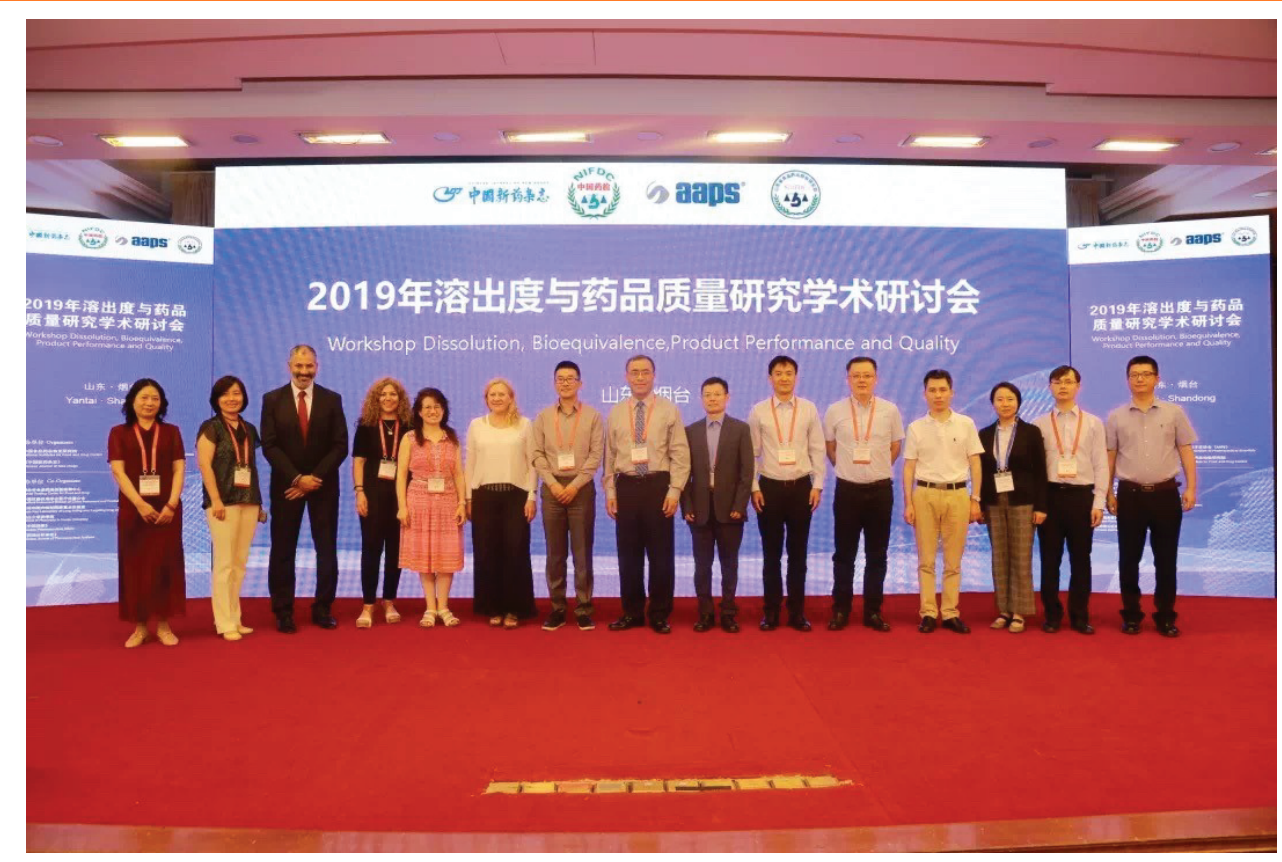

Figure 1. Workshop speakers and organizing committee members (left to right): Mrs. Ailing Zou, Yantai Institute for Food and Drug Control; Dr. Liping Zhou, Mr. Samir Haddouchi, Dr. Nikoletta Fotaki, Dr. Sandra Suarez Sharp, Dr. Diane Burgess, Dr. Zhen Liu, Dr. Xujin Lu, Dr. Baoming Ning, Mr. Mingzhe Xu, National Institutes for Food and Drug Control; Mr. Weijian Wang, Shandong Institute for Food and Drug Control; Mr. Yuwen Xu, Shandong Institute for Food and Drug Control; Ms. Jinlan Zhang, Institute of Materia Medica, Chinese Academy of Medical Sciences \& Peking Union Medical College; Dr. Chengyu Liu, Tsinghua University; and Mr. Yu Feng, Chinese Journal of New Drugs. 